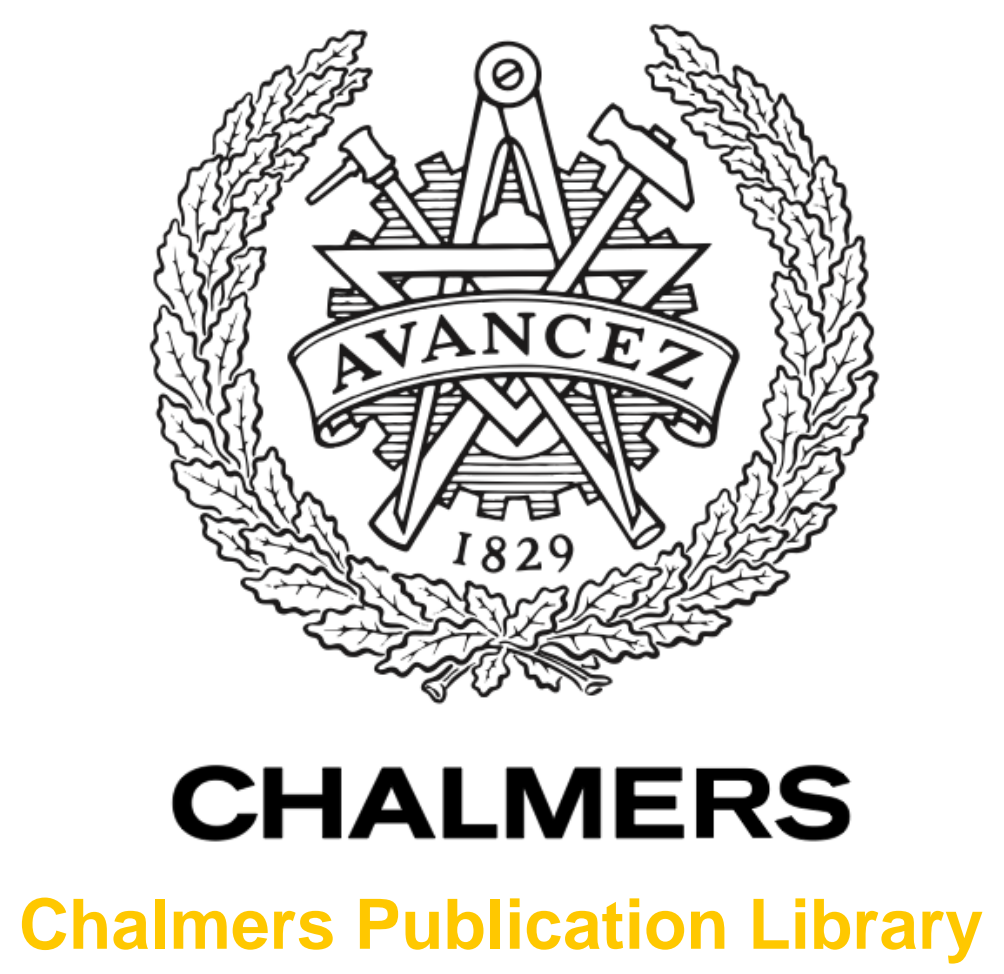

\title{
Planar Dual-Mode Horn Array with Corporate-Feed Network in Inverted Microstrip Gap Waveguide
}

This document has been downloaded from Chalmers Publication Library (CPL). It is the author's version of a work that was accepted for publication in:

IEEE Transactions on Antennas and Propagation (ISSN: 0018-926X)

Citation for the published paper:

Pucci, E. ; Rajo-Iglesias, E. ; Vazquez-Roy, J. (2014) "Planar Dual-Mode Horn Array with Corporate-Feed Network in Inverted Microstrip Gap Waveguide". IEEE Transactions on Antennas and Propagation, vol. 62(7), pp. 3534 - 3542.

http://dx.doi.org/10.1109/TAP.2014.2317496

Downloaded from: http://publications.lib.chalmers.se/publication/200969

Notice: Changes introduced as a result of publishing processes such as copy-editing and formatting may not be reflected in this document. For a definitive version of this work, please refer to the published source. Please note that access to the published version might require a subscription.

Chalmers Publication Library (CPL) offers the possibility of retrieving research publications produced at Chalmers University of Technology. It covers all types of publications: articles, dissertations, licentiate theses, masters theses, conference papers, reports etc. Since 2006 it is the official tool for Chalmers official publication statistics. To ensure that Chalmers research results are disseminated as widely as possible, an Open Access Policy has been adopted.

The CPL service is administrated and maintained by Chalmers Library. 


\title{
Planar Dual-Mode Horn Array with Corporate-Feed Network in Inverted Microstrip Gap Waveguide
}

\author{
Elena Pucci, Eva Rajo-Iglesias, Senior Member, IEEE, Jose-Luis Vazquez-Roy, Member, IEEE \\ and Per-Simon Kildal, Fellow, IEEE
}

\begin{abstract}
The gap waveguide technology was recently introduced as an alternative to hollow waveguides and Substrate Integrated Waveguides (SIWs) for mm-wave applications. This paper presents the design of a 4 by 4 planar dual-mode horn array with low loss corporate feed network realized by using an inverted microstrip gap waveguide. The dual-mode horns are compact and designed to reduce the power losses in grating lobes. It is because the diameters of the horn apertures are larger than two wavelengths to allow more space for the feed network and thereby lower conductive losses. The measurements show very good agreement with simulations, with $10 \%$ bandwidth of the return loss, $25 \mathrm{dBi}$ realized gain and about $60 \%$ aperture efficiency.
\end{abstract}

Index Terms-Gap waveguide, dual-mode horn, array, grating lobes, feed network.

\section{INTRODUCTION}

$\mathbf{T}$ HE growing interest towards millimeter wave applications like automotive, radar, communications system terminals, and space, has led to the need of planar antenna systems with low volume and cost. Microstrip antenna arrays are promising candidates for these applications because of their light weight and low production cost. However, they suffer from high losses in the feed network. Dielectric losses are a critical constraint for high gain antennas as shown in [1]. Dielectric losses are large for materials with high permittivity, and will also be generated by non-uniformities in the thin substrate material. Conductive losses become more significant the higher the frequency because then the feed lines are narrower. In addition, radiation from the feed network can cause high side lobe levels, as well as errors in the excitation of the elements [2], [3]. These problems become even more critical when handling arrays with large aperture.

The inverted microstrip gap waveguide feed network used in the present paper does not suffer from dielectric and radiation losses. The gap waveguide technology was proposed in [4] and [5]. There exist three different versions of gap waveguides: ridge, groove and microstrip gap waveguides [6], shown in Fig. 1. The ridge gap waveguide was validated in [7], the groove gap waveguide is good for designing high-Q filters [8], [9], and the inverted microstrip gap waveguide

E. Pucci and P.-S. Kildal are with the Department of Department of Signals and Systems, Chalmers University of Technology, Gothenburg, 41296 Sweden, e-mail: elena.pucci@chalmers.se; per-simon.kildal@chalmers.se.

E. Rajo-Iglesias and J.-L. Vazquez-Roy are with the Department of Signal Theory and Communications, University Carlos III of Madrid, Madrid, Spain. was demonstrated in [10] and [11]. The theoretical basis of the gap waveguide technology is illustrated schematically in Fig. 2. A parallel plate cut-off region is created in the air gap between two parallel plates, one of which is a smooth metal plate and the other a textured surface in the form of e.g. an Artificial Magnetic Conductor (AMC) at a distance smaller than quarter wavelength from the smooth metal plate. The two surfaces create together a stopband for the parallel-plate modes. Therefore, the field will only propagate along metal ridges, grooves or strips, placed in the textured surface. In all the other directions, the stopband prevents any leakage loss. The most common AMC used in gap waveguides is a metal pin surface, i.e. the so called 'bed of nails' [12]. Mushroom-type textured EBG structures as introduced by Sievenpiper [13] are instead used for low frequency applications because they are much more compact [11].

Arrays of horns fed by normal rectangular waveguides have low losses. However, they do not meet the demand of low profile and light weight that enables integration with electronic parts, at least not if the required bandwidth is so large that corporate feeding must be used. Rectangular waveguides must at high frequency be realized in two pieces that must be joined together with good electrical contact between them. This conducting joint makes them expensive to manufacture. The gap waveguides are realized in two parallel flat surfaces as well, but these do not need to be in metal contact, which is attractive from a manufacturing point of view.

Substrate Integrated Waveguides (SIWs) are realized in planar form by using printed circuit board and two rows of via holes embedded in the substrate [14], [15], and they work similar to conventional hollow waveguides. However, SIWs present larger losses at millimeter waves than hollow waveguides and gap waveguides due to the loss tangent of the substrate. Leakage losses are also an issue in SIW, because the structure is not perfectly shielded by the via holes [16], [17]. SIWs have been also used for array antennas. In [14] a simple single-layer feed waveguide composed of densely arrayed posts has been introduced. However, this design requires a grounded dielectric substrate for the via holes and the desired performances strongly depend on the posts position error which is difficult to control in practical realizations. Also, series-fed singlelayer waveguides are simple but have problems of narrow bandwidth due to their long lines [18]. A multilayer structure is instead developed in [19] to achieve wide bandwidth with high efficiency, but the multilayer solution requires a more 

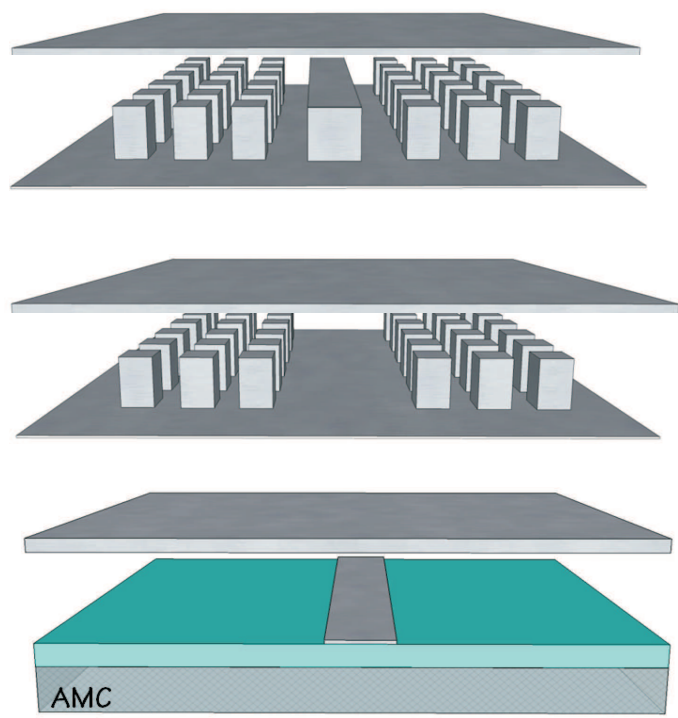

Fig. 1. From top to bottom: ridge, groove and microstrip gap waveguides.
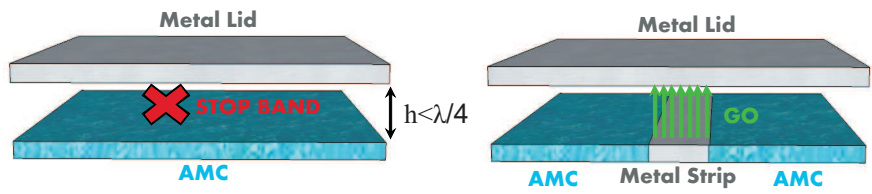

Fig. 2. Schematic of the stopband and transmission of the wave in gap waveguide technology.

complex design and manufacturing at high frequency.

The gap waveguide technology combines the benefits of waveguides (low losses, high Q-factors and can be made of only metal) with the benefits of microstrip/SIW (low profile and planar design), and there is no problem with surface waves and unwanted radiations as shown in [20], [21]. The low losses are documented by the study in [22].

The microstrip gap waveguide is very similar to inverted also called suspended microstrip lines, because the cutoff between the smooth metal surface and the textured surface will force the field to travel in the air gap between the metal strip and the upper smooth surface. We therefore also call it an inverted microstrip gap waveguide. The benefit of this solution is that a uniform bed of nails (or other type of AMCs) can be used below the microstrip line, making manufacturing easier and cheaper. E.g., a uniform periodic surface can be sawed with parallel saw blades, whereas non-uniform pin locations and ridges must be milled with a thin milling tool. For these reasons, the microstrip gap waveguide is attractive in feed networks for slot antenna arrays at high frequency.

This paper will present the design of a sixteen element planar dual-mode horn array fed by an inverted microstrip gap waveguide corporate distribution network. The final antenna is intended for $60 \mathrm{GHz}$ applications, however, the prototype shown in this paper is realized at $10 \mathrm{GHz}$ as a first step. Other types of gap waveguide antennas have been also recently developed. A multi-layer phased array in gap waveguide technology has been realized for $76 \mathrm{GHz}$ in [23]. The multi-layer solution has as a drawback a more complicated design compared to the single-layer array. A four-element planar slot array excited by a ridge gap waveguide single-layer corporate feed network is then developed in [24]. However, the realization of this design at high frequency is more difficult because of non-uniform pin locations around the feed network and a very thin milling tool would be needed to realize the pins and the ridges.

The design presented in the present paper is a sixteen-element planar horn array excited by a microstrip gap waveguide single-layer corporate feed network. It will be shown that such feed network has wide lines, thus reducing tolerances problems and being low loss; it is easy and cheap to realize, by using standard PCB boards and uniform bed of nails. It will also be shown that this feed network does not have problems of coupling between the wide lines and no problems with surface waves that can be generated from the substrate, as the field is traveling in the air. For these reasons, the microstrip gap waveguide is seen as an advantageous solution for high frequency array antennas.

\section{Characteristics of The Dual-Mode Horn ELEMENT}

The design of the single dual-mode horn element is based on the initial study performed in [25]. The presented inverted microstrip gap waveguide feed network takes more space than conventional microstrip feed networks. The reason is that we want to use wider lines to reduce the conductive losses, and we can in gap waveguides use wider lines than in microstrip lines. Also, we have no problems with leakage in terms of surface waves and radiation, which are presented in normal microstrip networks. This implies that the distance between the horn elements will be larger than a wavelength, so that grating lobes will appear along the principal planes. Therefore, directive $2 \lambda$ by $2 \lambda$ square horns are used in order to reduce the power losses in the grating lobes. The goal is to have horn antenna elements with a nearly uniform aperture distribution, so that the first grating lobe will ideally fall in the first null of the individual element radiation pattern. Initially, we perform the electromagnetic design of the single isolated element, because directive horns are uncoupled when placed side by side. The geometry of the horn element is shown in Fig. 3 . The design consists, from bottom to top, of a uniform grid of metal pins below the microstrip feed line forcing the field to travel in the air gap $h=1.5 \mathrm{~mm}$, exciting the slot on the upper lid, which has length $L=15.96 \mathrm{~mm}$ and width $W=8.82$ $\mathrm{mm}$. The field will radiate through the dual-mode horn. One constraint in the design is the height of the horn, which must be small to keep the desirable as-planar-as-possible profile. Thus, we choose $h_{1}=30 \mathrm{~mm}$, which is about one wavelength at the frequency of operation. The dual-mode characteristic is obtained by introducing a step in the H-plane, of length $L_{1}=21.36 \mathrm{~mm}$, to allow the excitation of the $\mathrm{TE}_{30}$ mode, which will combine with the dominant $\mathrm{TE}_{10}$ mode to a quasiuniform distribution [26, Sec.8.3], given that the $\mathrm{TE}_{10}$ and $\mathrm{TE}_{30}$ modes have opposite phase at the horn aperture. The phase variation of the total field over the horn aperture is smaller for longer horns, but in our case we need to find a 

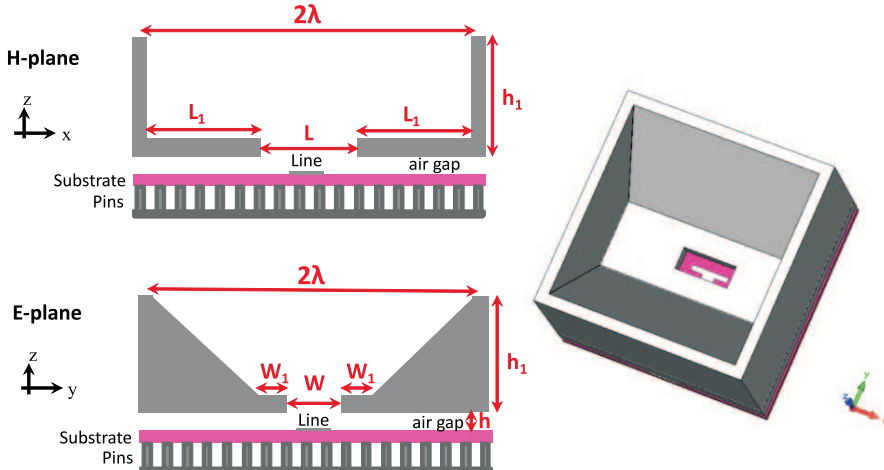

Fig. 3. Dual-mode horn element fed by microstrip gap waveguide and 2D views in E- and H-planes. The following dimensions are used: $h=1.5 \mathrm{~mm}$, $L=15.96 \mathrm{~mm}, W=8.82 \mathrm{~mm}, h_{1}=30 \mathrm{~mm}, L_{1}=21.36 \mathrm{~mm}$ and $W_{1}=6$ $\mathrm{mm}$. The horn aperture has dimensions of $58.68 \times 58.68 \mathrm{~mm}$.

trade-off between this phase variation and the length of the horn to keep a planar design. The final far field functions of the horn element in E- and H-planes are shown in Fig. 4. The first grating lobe appears at the directions

$$
\theta= \pm \sin ^{-1}(\lambda / d)
$$

from broadside, in the principal planes, where $d$ is the element spacing. The level of this grating lobe relative to the broadside main lobe can be directly extracted from the element far field function at these directions. Fig. 5 shows the reduction of the grating lobe level in E- and H-plane for the dual-mode proposed design compared to the standard horn also made in the same gap waveguide technology.

The antenna element has also been simulated in the presence of the other elements by using an infinite periodic extension of the antenna unit cell in CST Microwave Studio [27]. The element pattern is then multiplied by the array factor to obtain the far field pattern for the antenna array built of sixteen identical elements. These far field patterns are presented in Fig. 6 in E- and H-plane showing the grating lobes appearing as predicted. The corresponding grating lobe efficiency (i.e. the reduction of the directivity due to the grating lobes) is presented in Fig. 7 and can be calculated by using the following formula presented in [28, Sec.10.3.5]:

$$
e_{\text {grating }}=\left|G\left(\theta_{\mathrm{o}}, \varphi_{\mathrm{o}}\right)\right|^{2} /\left\{\sum_{p q}\left|G\left(\theta_{p q}, \varphi_{p q}\right)\right|^{2} \frac{\cos \theta_{\mathrm{o}}}{\cos \theta_{p q}}\right\},
$$

defined as the reduction of the directivity (or aperture efficiency) of the array due to the sum of all visible grating lobes.

We would like to have a considerable bandwidth, at least $10 \%$ at the frequency of operation. For this reason, a T-shaped feed line section, shown in Fig. 8, is used to obtain about $10 \%$ matching bandwidth [29], with $50 \Omega$ line impedance, as presented from the simulated $\mathrm{S}_{11}$ in Fig. 9(a). Fig. 9(b) shows also the simulated realized gain of the horn element with pins underneath.

The distribution network was first designed numerically with an ideal PMC condition located at the top of the pins [30], in order to reduce computation time during optimization. There

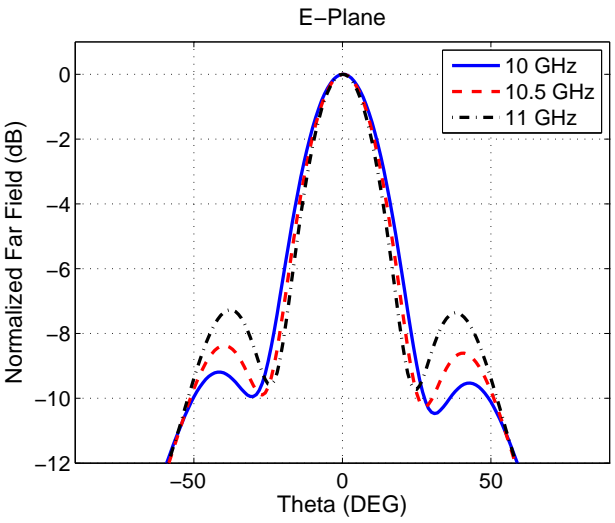

(a)

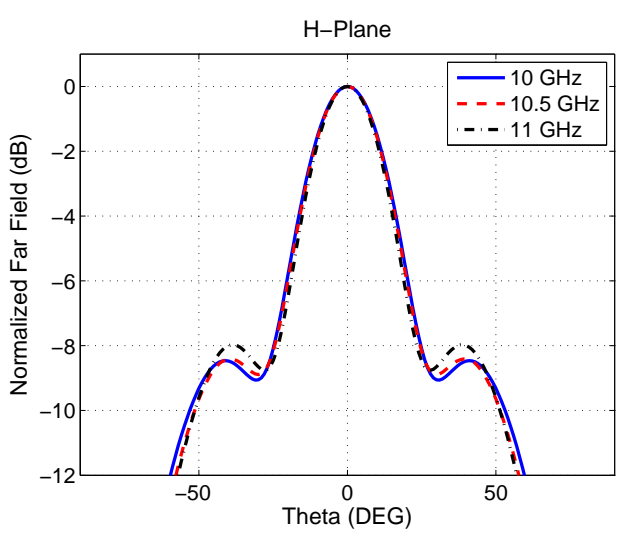

(b)

Fig. 4. Element far field radiation patterns in (a) E-plane and (b) H-plane.

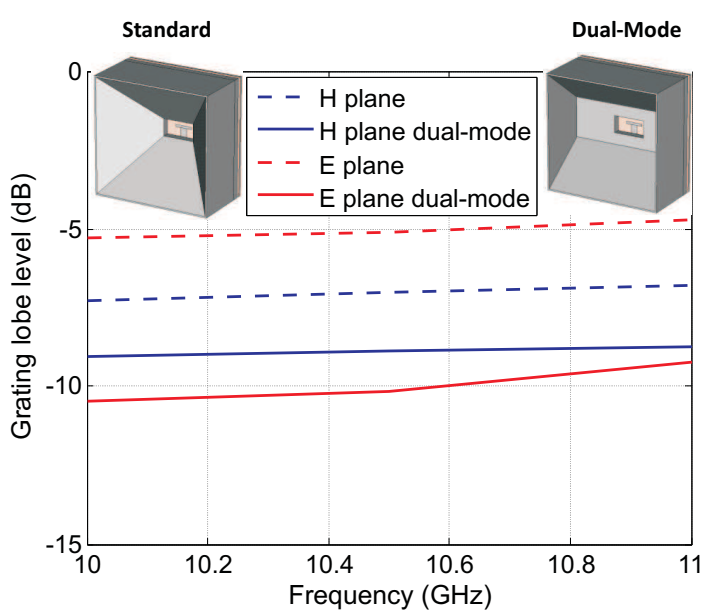

Fig. 5. Grating lobe level as a function of frequency for the proposed design (continuous lines) and the standard horn (dashed lines) in E- and H-planes.

was a slight shift upwards in frequency when the bed of nails was added, as seen from the plot. This shift in frequency is due to a change of the characteristic impedance of the microstrip line when it is placed above the pins. In [10] the effect of the pins on the characteristic impedance is numerically studied and compared to the ideal case of PMC condition. This study showed that $Z_{o}$ for a suspended microstrip gap waveguide with bed of nails is smaller than the $Z_{o}$ of an ideal gap 


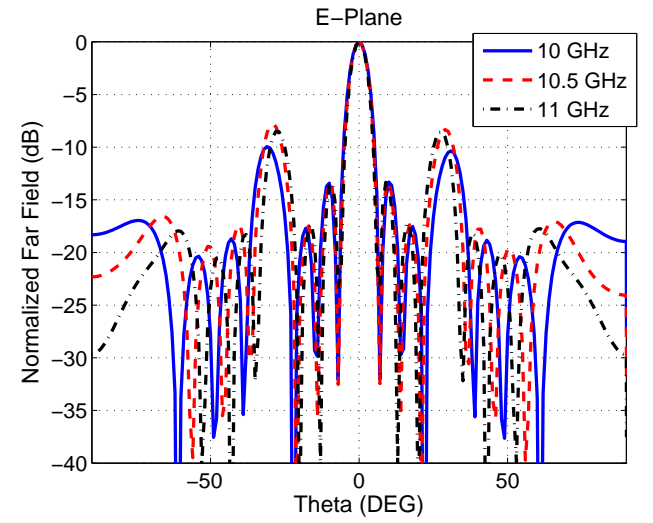

(a)

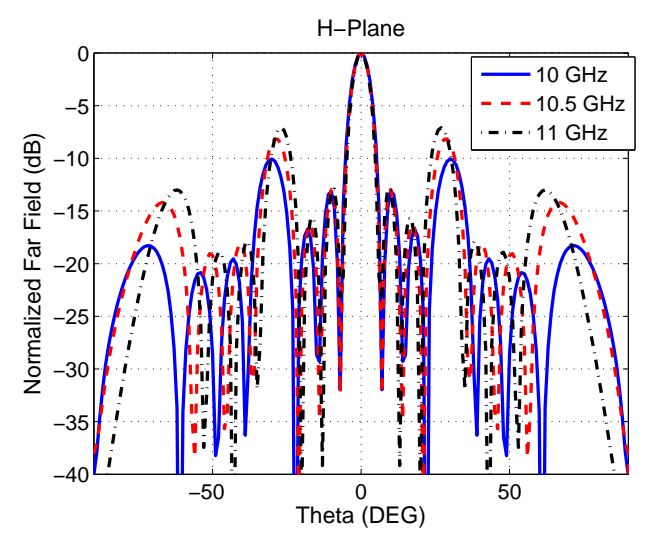

(b)

Fig. 6. Simulated radiation patterns of the periodic extension of the antenna element in (a) E-plane and (b) H-plane.

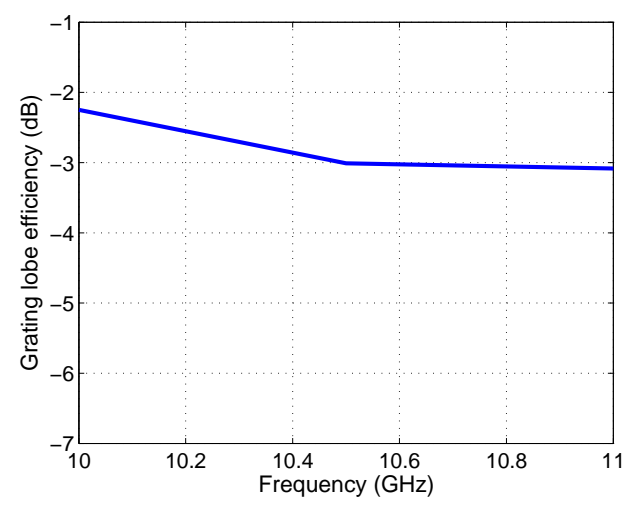

Fig. 7. Aperture efficiency of the horn array due to grating lobes as a function of frequency calculated for the far field functions in Fig. 6 .

waveguide (with metallic strip resting above PMC plate). In spite of the difference between pins and ideal PMC, the PMC is a good starting point because the numerical simulations are so much faster. The dimensions chosen for the pins are shown in Fig. 10 providing a stopband from $8.5 \mathrm{GHz}$ to $13 \mathrm{GHz}$. The dispersion diagram is plotted for an infinite periodic unit cell. The dielectric material used is Rogers RO 3003 with permittivity equal to $3, \tan \delta=0.0013$ and thickness $1.5 \mathrm{~mm}$.

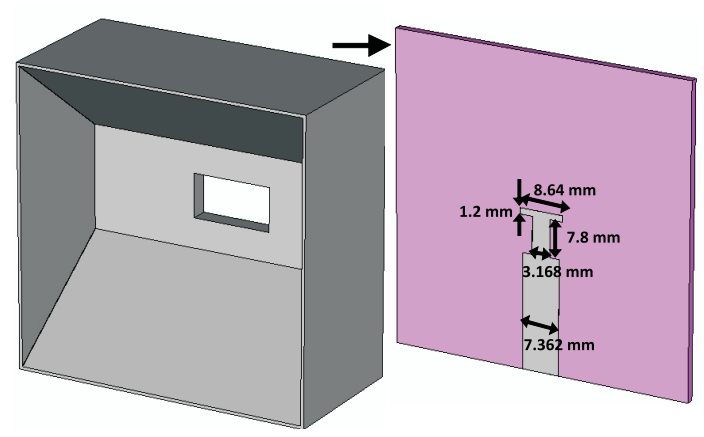

Fig. 8. Dual-mode horn element fed by T-shaped feed line with its dimensions.

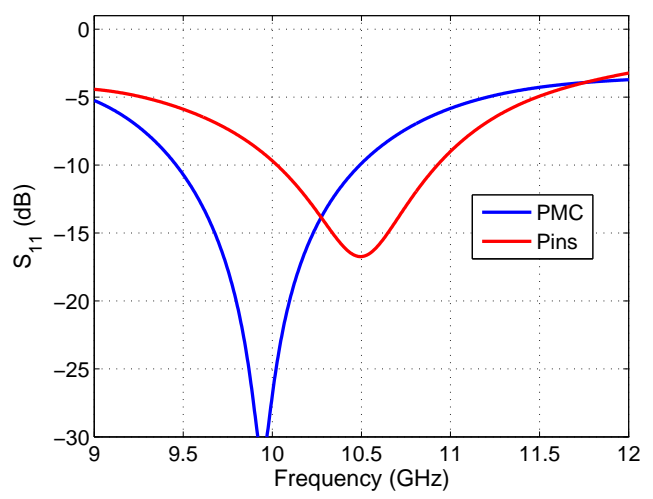

(a)

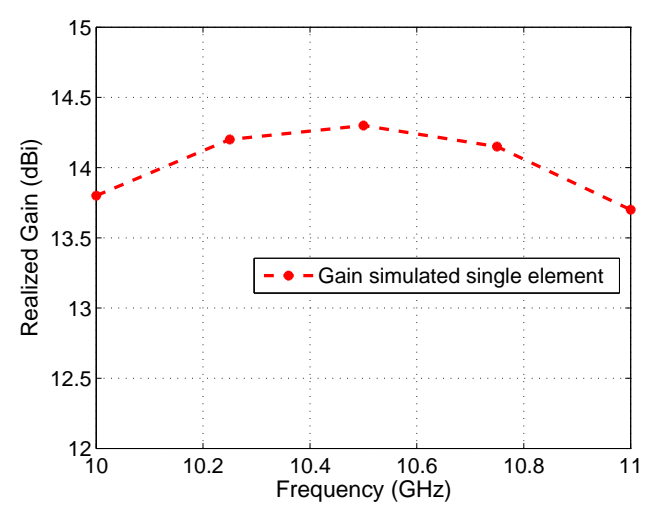

(b)

Fig. 9. (a) Simulated $\mathrm{S}_{11}$ for the dual-mode horn element with ideal PMC and bed of nails under the substrate. (b) Simulated realized gain for the dual-mode horn element with bed of nails.

\section{Corporate Feed Network}

The array elements are fed by a corporate feed network realized in microstrip gap waveguide. The feed network is used to split the power with equal amplitude and phase to the radiating elements by using T-shaped power dividers and quarter wavelength impedance transformers. The geometry of the 4 by 4 corporate feed network is shown in Fig. 11. The input is provided from the central $50 \mathrm{Ohms}$ transmission line, which splits the power into two main branches. Then, the next two power dividers (one above and one below) are used to equally divide the wave along each of the four smaller feed networks, designed in such a way that the power is divided 


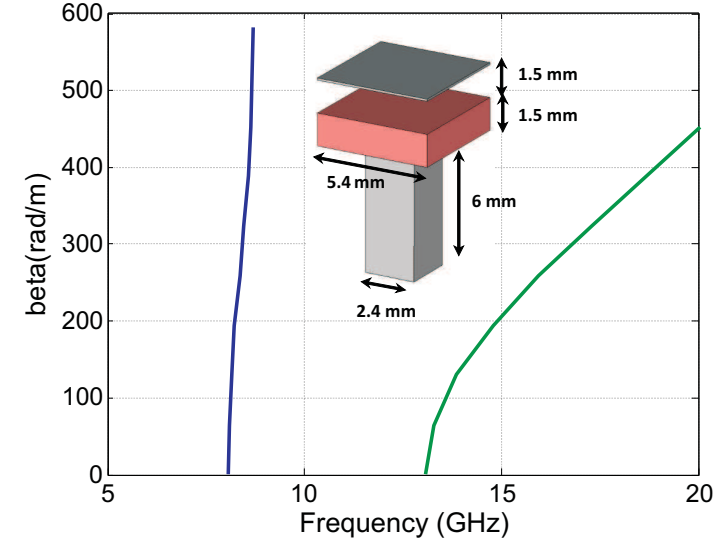

Fig. 10. Dispersion diagram for an infinite periodic unit cell with a pin of thickness $2.4 \mathrm{~mm}$ and length $6 \mathrm{~mm}$. The period is seen to be $5.4 \mathrm{~mm}$ and the air gap $1.5 \mathrm{~mm}$.

with equal phase at each of the four radiators. The matching is obtained by using impedance transformers and by mitering the corners of the bends. The dimensions of the power splitter that is closest to the radiating elements are shown in the layout in Fig. 11(b).

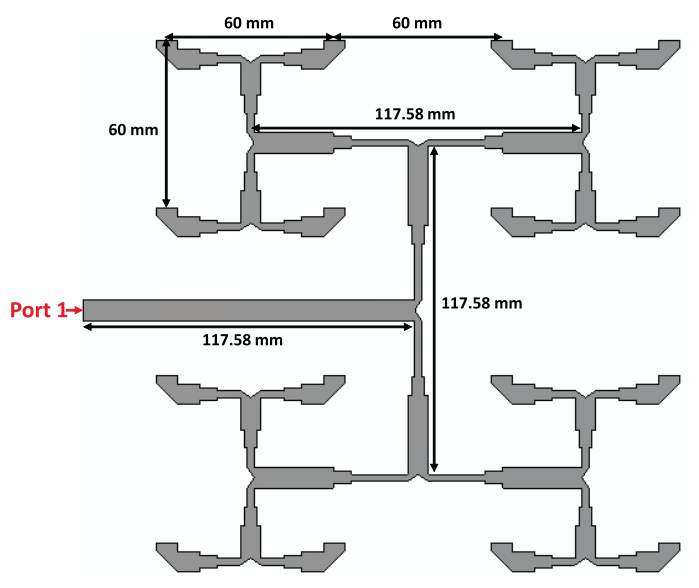

(a)

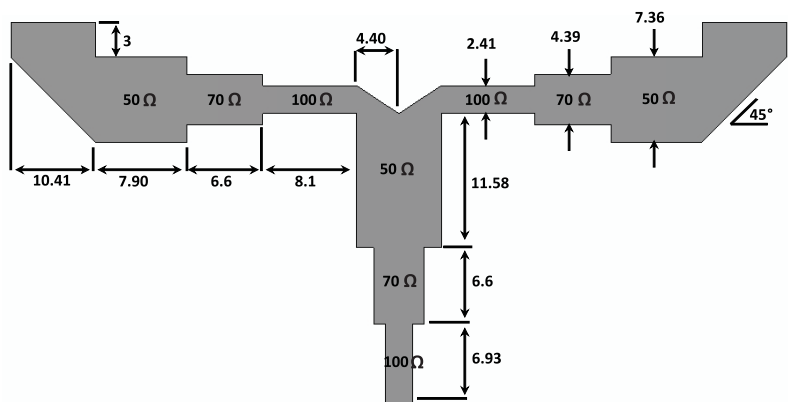

(b)

Fig. 11. (a) 4 by 4 corporate feed network and (b) layout of the power dividers at the radiating elements (dimensions are expressed in $\mathrm{mm}$ ).

The parameters are first designed in ADS [31] and then optimized using full-wave analysis with CST Microwave Studio [27]. The field is propagating in the air gap, so free space wavelength is used for the initial ADS design. Fig. 12(a) and 12(b) show the final simulated S-parameters in CST for the
2 by 2 and for the 4 by 4 feed networks, respectively. The power distribution between outputs is seen to be good for both cases. The frequency ranges for which $\mathrm{S}_{11}$ is below $-10 \mathrm{~dB}$ are $9.4-11.6 \mathrm{GHz}$ and $10.2-11.2 \mathrm{GHz}$ for the two cases, corresponding to $21 \%$ and $10 \%$ bandwidths, respectively. The plots present also the $\mathrm{S}_{11}$ of the feed networks above an ideal $\mathrm{PMC}$, showing that the presence of pins is affecting the results much more than in the study done in [10], probably due to the more complicated circuit. The simulated insertion loss, without mismatch factor, for the 4 by 4 feed network with pins and ideal PMC is presented in Fig. 13. The simulation with pins in one case also includes a thin layer of foam material as air gap, which will be used in the realized prototype (in Section IV), with permittivity $\epsilon=1.08$ and $\tan \delta=0.0021$ at $10 \mathrm{GHz}$. The results are shown for different situations: the feed network with ideal PMC and lossless strip lines and substrate ('PMC lossless strips and substrate'), with lossless strip lines and lossy substrate ('PMC lossless strips'), with lossy strip lines and substrate ('PMC'), and the latter is done also with real pins without foam and with foam in the air gap ('Pins' and 'Pins foam', respectively). The foam material adds about $0.5 \mathrm{~dB}$ loss to the feed network due to its tangent loss. On the other hand, the difference between PMC with lossless materials and real materials is very little, about $0.1 \mathrm{~dB}$ between each other. The pins case without foam has about $0.5 \mathrm{~dB}$ loss in its frequency range of operation, which is above $10 \mathrm{GHz}$.

\section{Simulated \& Measured Results for the Array}

The three layers of the whole sixteen element horn array antenna are shown in Fig. 14. The horn layer is separated from the corporate feed network by a $1.5 \mathrm{~mm}$ air gap. The feed network is resting over a uniform grid of nails. The total size of the array is $8 \lambda$ by $8 \lambda$, which corresponds to $24 \times 24$ $\mathrm{cm}^{2}$. The distance between each slot element is about $2 \lambda$, i.e., $60 \mathrm{~mm}$. The horns are placed side by side and the dimensions of each dual-mode horn are shown in Fig. 3. The nails layer is realized by milling several rows of metallic pins in a metal plate of dimensions $24 \mathrm{~cm} \times 24 \mathrm{~cm}$. The pins have width 2.4 $\mathrm{mm}$, height $6 \mathrm{~mm}$ and period $5.4 \mathrm{~mm}$, as shown in the sketch in Fig. 15.

The realized prototype is shown in Fig. 16. The corporate feed network is placed above the bed of nails, and the thin layer of Rohacell HF51 foam (1.5 mm thick) is used to pressure the corporate feed network on to the top of the pins. The foam material has a permittivity very close to 1 , thereby also defining the air gap needed for the fields to propagate along the feed network. The input antenna port is provided from the side by using an SMA connector with a flat inner conductor. This is pressured (and not soldered) between the foam layer and the microstrip line when the whole horn array is integrated. The upper metal layer contains the dual-mode horns excited by slots, and it is connected to the lower pins layer by screws at each of the four corners, as shown in the figure. The metal used is Aluminium. The feed network is realized by ordinary printed circuit technology on PCB, therefore the tolerances are the same as for regular microstrip lines, which are already well established. Even more in this 


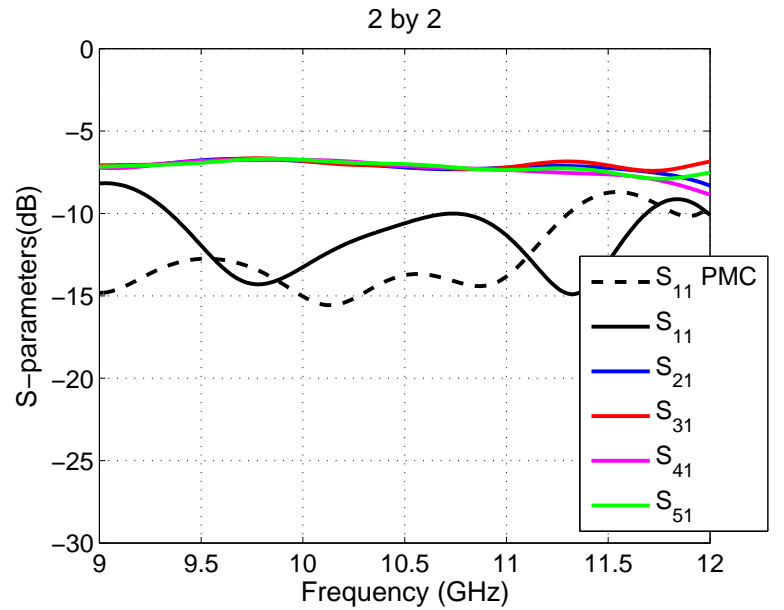

(a)

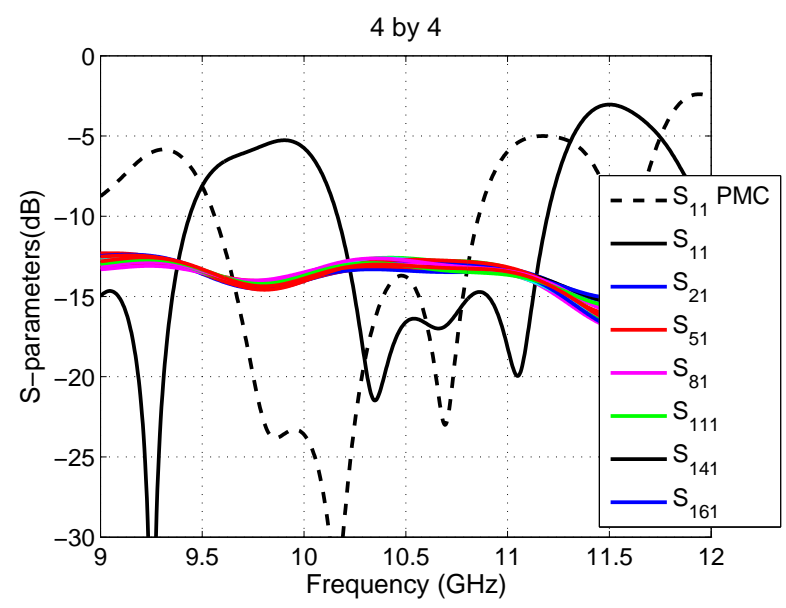

(b)

Fig. 12. Simulated S-parameters obtained from CST for the (a) 2 by 2 and (b) 4 by 4 corporate feed network with bed of nails compared with the ideal PMC case.

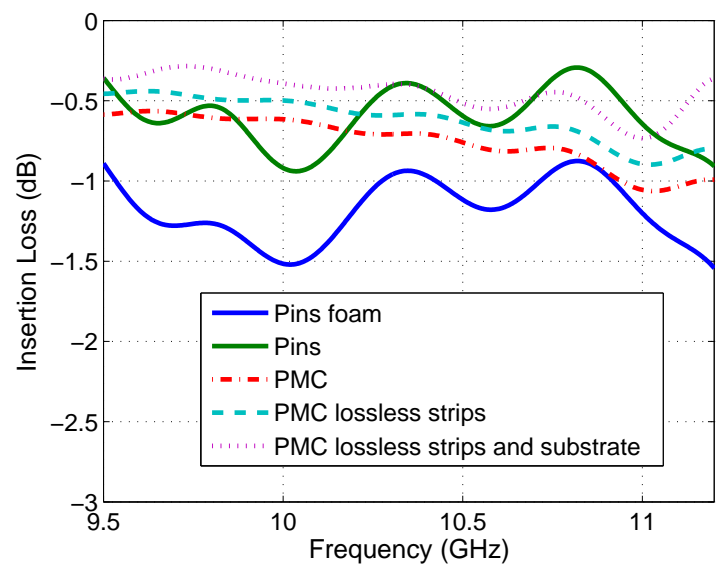

Fig. 13. Simulated insertion loss without mismatch factor for the feed network with pins and with PMC. The results are presented for different cases, according to the legend from bottom to top: PMC with lossless strip lines and substrate, with lossless strip lines and lossy substrate, with lossy strip lines and substrate, and the latter is done also with real pins without foam and with foam in the air gap.
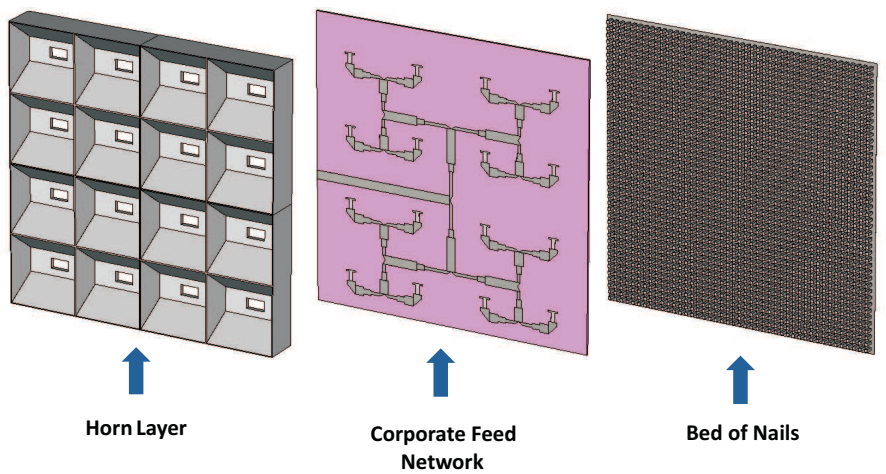

Fig. 14. Sixteen element array design. From top to bottom: horn array, corporate feed network and grid of pins. There is an air gap of $1.5 \mathrm{~mm}$ dividing the horns and the feed network.

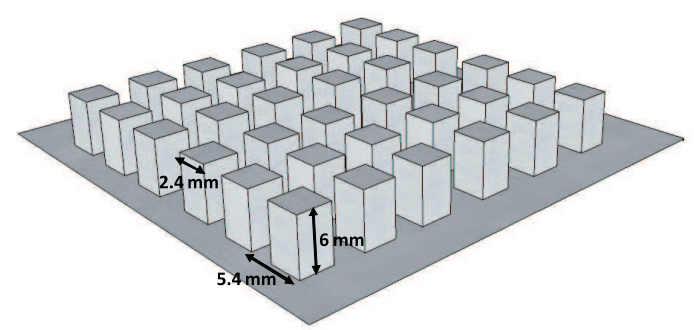

Fig. 15. Sketch of a bed of nails with pins dimensions used for the array antenna.

case the tolerances are less strict, as the lines are wider than in microstrip lines in substrates. With respect to the pin structures, they are wide band, therefore it is not very critical neither pins dimensions, nor the gap size. The antenna block has been realized by wire cut and $\mathrm{CNC}$ machining technique. The simulated and measured $\mathrm{S}_{11}$ is presented in Fig. 17. About $11 \%$ bandwidth is obtained with an $\mathrm{S}_{11}$ below -10 $\mathrm{dB}$ from 10 to $11.16 \mathrm{GHz}$. There is good agreement with the simulated results, even though we experienced that the matching is very sensitive to the quality of the pressure contact between the SMA and the feed line. The plot also shows the simulated $\mathrm{S}_{11}$ of the array when the PMC condition is used below the feed network instead of the pins. The PMC case moves down in frequency.

The far-field patterns in E- and H-planes and in the 45deg plane have been measured in an anechoic chamber. The simulated and measured patterns are shown in Fig. 18. They agree very well, showing the first grating lobes appearing when $\theta$ is about $30^{\circ}$, i.e., the same that was predicted from the single element study and equation (1) in Section II, with a distance between the slots of $2 \lambda$. Indeed, the grating lobe levels in Eand $\mathrm{H}$-plane agree with the ones of the embedded element pattern in Fig. 6, being about $-10 \mathrm{~dB}$ in E-plane and $-8 \mathrm{~dB}$ in H-plane, and increasing with frequency. On the other hand, the grating lobes in the 45-deg plane are lower, being the product (sum of $\mathrm{dB}$ values) of the grating lobe levels in Eand $\mathrm{H}$-plane. The good agreement between simulations and measurements shows that we have good numerical control of the design, which will be useful also at higher frequency.

The power loss due to the grating lobes is an important 


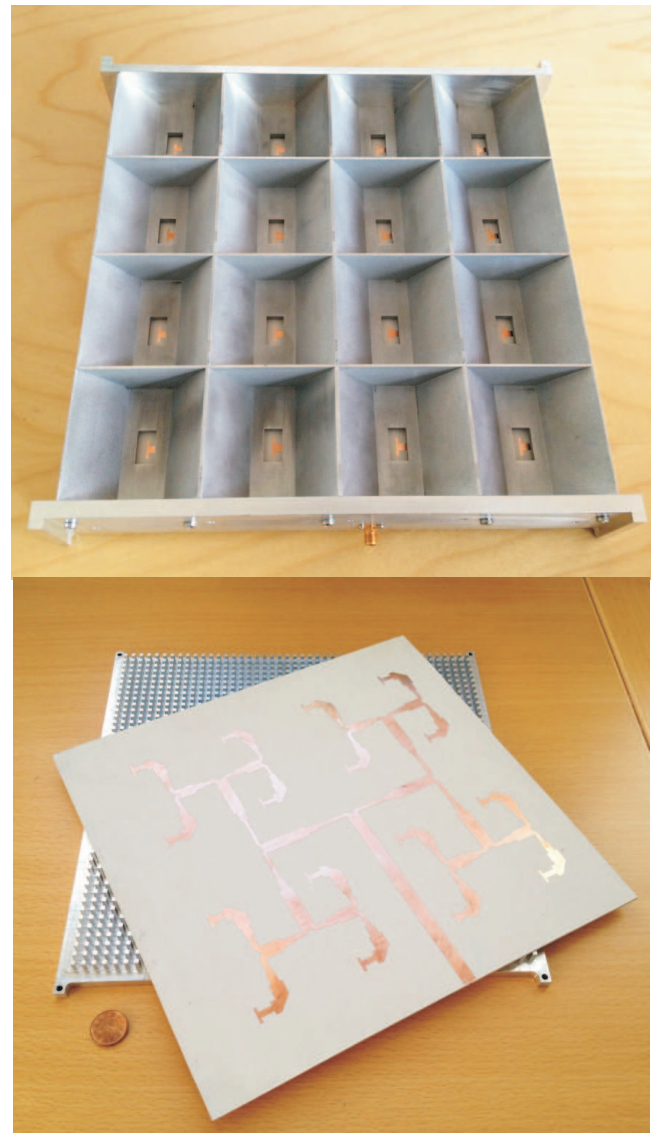

Fig. 16. Realized hardware of the $4 \times 4$ horn array: bed of nails, corporate feed network (bottom figure) and horn layer (upper figure). The foam layer above the feed network is not shown.

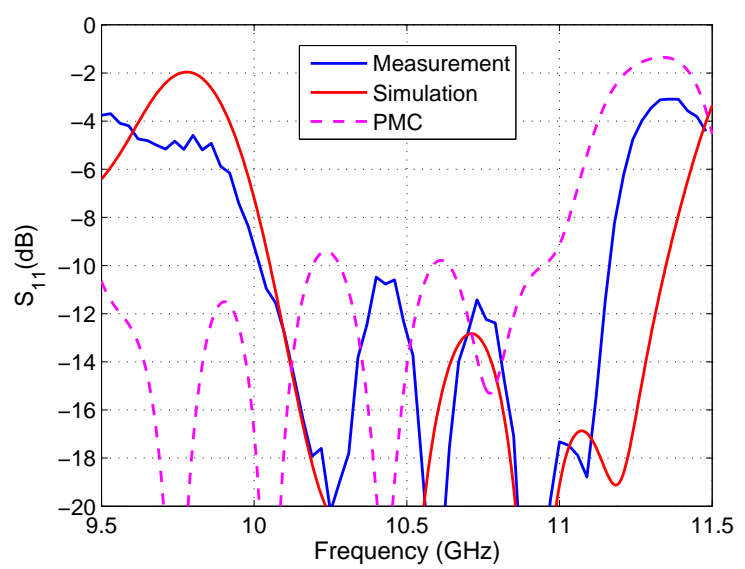

Fig. 17. Simulated and measured $\mathrm{S}_{11}$ for the sixteen elements dual-mode horn array.

parameter for the array design. The aperture efficiency can be computed from the simulated directivity as

$$
e_{\text {grating }}=D / D_{\max },
$$

where $D_{\max }=4 \pi A / \lambda^{2}$, with A the physical area of the array aperture, i.e. the periodic unit cell area times 16 . The grating lobe efficiency in (2) has been calculated for both simulated and measured far-field patterns of the array and compared with

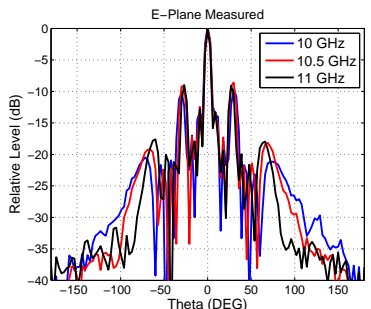

(a)

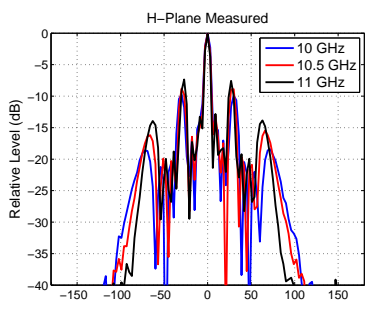

(c)

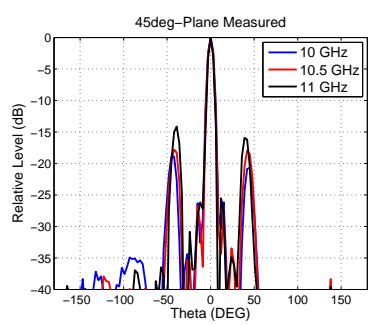

(e)

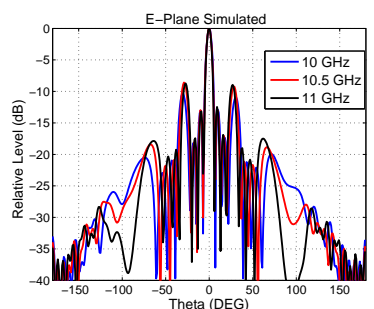

(b)

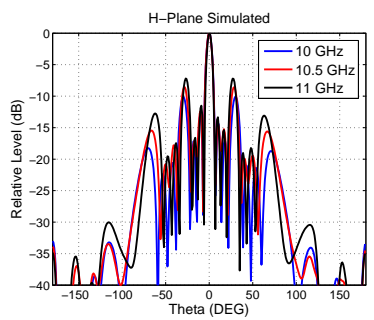

(d)

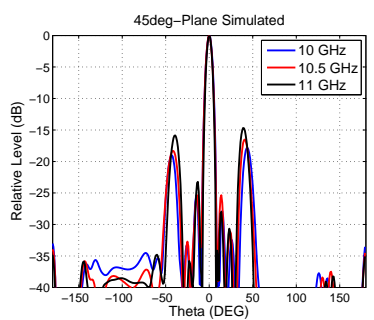

(f)
Fig. 18. Measured and simulated far-field radiation patterns in E- and $\mathrm{H}$ plane, and 45-deg plane.

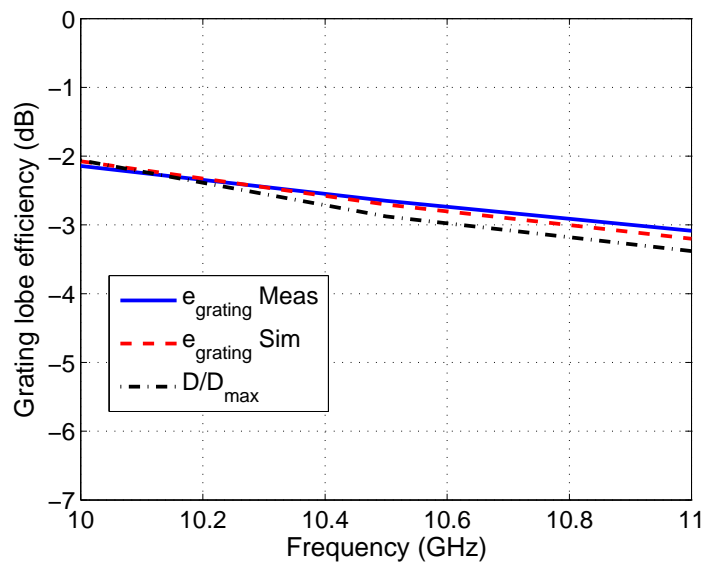

Fig. 19. Grating lobe efficiency calculated from eqn (2) for the simulated and measured far field patterns, and for comparison the computed aperture efficiency $D / D_{\max }$.

the aperture efficiency calculated by (3) from simulations. The results in Fig. 19 show very good agreement between the different approaches, with a power loss varying from 2 to $3 \mathrm{~dB}$ as a function of frequency. From this it is clear that the power distribution network works well without creating amplitude and phase errors that reduce directivity further. The low aperture efficiency is entirely due to the grating lobes.

The realized gain has also been measured in an anechoic 


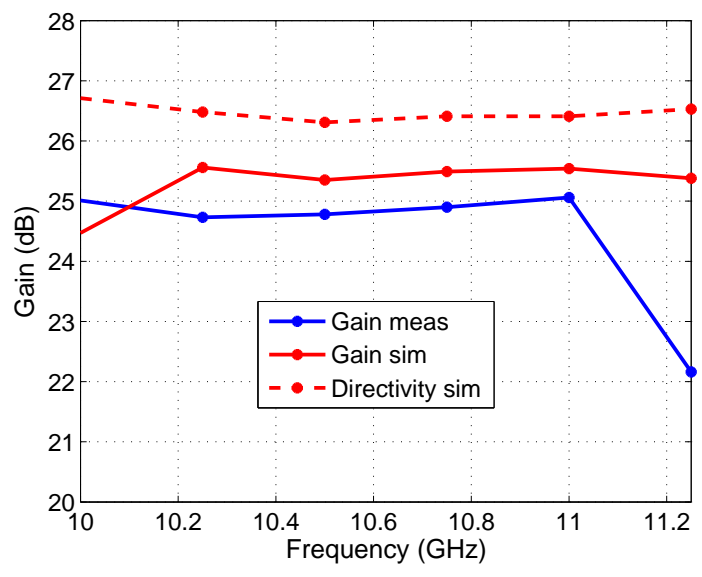

Fig. 20. Measured realized gain compared with simulated gain and directivity. The simulations were done including the foam in the air gap.

chamber. The measured realized gain compared with the simulated one and with the simulated directivity is presented in Fig. 20. The difference between simulated gain and directivity can be addressed to the mismatch and to the losses in the feed network and in the foam material. The simulations were done when the whole antenna was excited directly at its input inverted microstrip gap waveguide by a numerical waveguide port, in the same way as when exciting normal microstrip lines, whereas the measurements were done with coaxial connectors as explained above. Therefore, losses in the SMA connector as well as measurement uncertainty explain the difference between the simulated and the measured realized gains. We made additional simulations in order to explain the difference between measured realized gain and simulated directivity, and in particular we made simulations both with and without the foam material in the air gap. Fig. 21 shows the measured total radiation efficiency (' $e_{\text {totrad }}$ meas' calculated as $\left.\mathrm{G}_{\text {measured }} / \mathrm{D}_{\text {simulated }}\right)$, the computed total radiation efficiency ('e totrad $\operatorname{sim}$ '), and the computed radiation efficiency (i.e. excluding the mismatch factor) with and without foam material ('e $e_{\text {rad }}$ sim' and ' $e_{\text {rad }}$ sim no foam', respectively). The latter two cases are shown with continuous red and black curves. We see that the foam material adds about $0.5 \mathrm{~dB}$ loss to the circuit, as it was also seen in Section III from the simulated insertion loss of the only feed network shown in Fig. 13. Therefore, the loss due to the feed network is about half, in the order of $0.5 \mathrm{~dB}$ or less if we can manage without the foam material. There is about $0.5 \mathrm{~dB}$ difference between the measured and simulated total radiation efficiencies, which is attributed to SMA connectors, to impurities of the materials used in the circuit, and to the accuracy of the measurement, as already explained. In particular, this discrepancy is also due to the difference in mismatch factors between simulation and measurements, more visible at around $11.2 \mathrm{GHz}$, where the measured mismatch loss is very large for the measured $S_{11}$ (see Fig. 17).

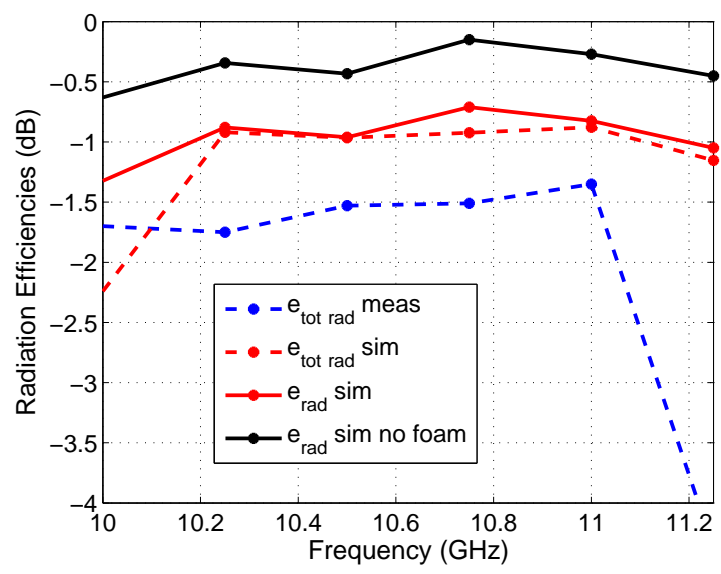

Fig. 21. Comparisons of simulated and measured total radiation efficiencies and simulated radiation efficiencies with and without foam material.

\section{CONClusion}

We have presented a sixteen element planar dual-mode horn array fed by inverted microstrip gap waveguide distribution network that has been designed and measured for operation in the frequency band 10-11.2 GHz. Compact dual-mode horns, one wavelength long and with an aperture of $2 \lambda$ by $2 \lambda$ are used to reduce the power loss due to the visible grating lobes. The spacious feed network has small conductive losses of about $-1.0 \mathrm{~dB}$, of which half is due to the foam material between the substrate and the upper smooth metal surface. The measured and simulated results agree quite well, showing an aperture efficiency of about $-2.5 \mathrm{~dB}$ at the mid frequency and a measured realized gain of about $25 \mathrm{dBi}$. The low aperture efficiency is due to grating lobes. In this case a compromise between low-profile design and grating lobe level was found, because longer horns will give narrower element patterns and hence lower grating lobes. The corporate inverted microstrip gap waveguide feed network is simple with a substrate over a uniform grid of bed of nails, providing a low loss structure. It is not easy to connect to the inverted microstrip line from other transmission lines. In the present work we chose a pressure contact between a coaxial connector with a flat center conductor, which could be done because we are operating at $10 \mathrm{GHz}$. The work shows that it is possible to make planar high gain antennas at $10 \mathrm{GHz}$ using inverted microstrip gap waveguide. In order to be successful also at higher frequency, an effective transition to e.g. standard rectangular waveguide should be developed. One such transition is reported in [32]. However, the waveguide is mounted to the smooth metal plate, i.e. to the radiating side of our array, so we could not use it. It is much more difficult to couple to waveguide aperture in between the pins of the bed of nails, for the inverted microstrip case.

\section{REFERENCES}

[1] J. Hirokawa and M. Ando, "Efficiency of $76 \mathrm{GHz}$ post-wall waveguidefed parallel-plate slot arrays," IEEE Transactions on Antennas and Propagation, vol. 48, no. 11, pp. 1742-1745, 2000. 
[2] R. J. Mailloux, J. F. McIlvenna, and N. Kernweis, "Microstrip array technology," IEEE Transactions on Antennas and Propagation, vol. 29 no. 1, pp. 25-37, 1981.

[3] E. Levine, G. Malamud, S. Shtrikman, and D. Treves, "A study of microstrip array antennas with the feed network," IEEE Transactions on Antennas and Propagation, vol. 37, no. 4, pp. 426-434, 1989.

[4] P. S. Kildal, E. Alfonso, A. Valero-Nogueira, and E. Rajo-Iglesias, "Local metamaterial-based waveguides in gaps between parallel metal plates," IEEE Antennas and Wireless Propagation Letters, vol. 8, pp. 84-87, 2009.

[5] P. S. Kildal, "Waveguides and transmission lines in gaps between parallel conducting surfaces," European patent application No. PCT/EP2009/057743, 2009.

[6] _ - "Three metamaterial-based gap waveguides between parallel metal plates for mm/submm waves," in 3rd European Conference on Antennas and Propagation (EuCAP 2009), pp. 28-32.

[7] P. S. Kildal, A. U. Zaman, E. Rajo-Iglesias, E. Alfonso, and A. ValeroNogueira, "Design and experimental verification of ridge gap waveguide in bed of nails for parallel-plate mode suppression," IET Microwaves, Antennas and Propagation, vol. 5, no. 3, pp. 262-270, 2011.

[8] A. U. Zaman, P. S. Kildal, and A. A. Kishk, "Narrow-band microwave filter using high-Q groove gap waveguide resonators with manufacturing flexibility and no sidewalls," IEEE Transactions on Components, Packaging and Manufacturing Technology, vol. 2, no. 11, pp. 1882-1889, 2012.

[9] E. Alfonso, A. U. Zaman, and P. S. Kildal, "Ka-band gap waveguide coupled-resonator filter for radio link diplexer application," IEEE Transactions on Components, Packaging and Manufacturing Technology, vol. 3, no. 99, 2013.

[10] A. Valero-Nogueira, M. Baquero, J. I. Herranz, J. Domenech, E. Alfonso, and A. Vila, "Gap waveguides using a suspended strip on a bed of nails," IEEE Antennas and Wireless Propagation Letters, vol. 10, pp. 1006-1009, 2011.

[11] E. Pucci, E. Rajo-Iglesias, and P. S. Kildal, "New microstrip gap waveguide on mushroom-type ebg for packaging of microwave components," IEEE Microwave and Wireless Components Letters, vol. 22, no. 3, pp. 129-131, 2012.

[12] M. G. Silveirinha, C. A. Fernandes, and J. R. Costa, "Electromagnetic characterization of textured surfaces formed by metallic pins," IEEE Transactions on Antennas and Propagation, vol. 56, no. 2, pp. 405415, 2008.

[13] D. Sievenpiper, Z. Lijun, R. F. J. Broas, N. G. Alexopolous, and E. Yablonovitch, "High-impedance electromagnetic surfaces with a forbidden frequency band," IEEE Transactions on Microwave Theory and Techniques, vol. 47, no. 11, pp. 2059-2074, 1999.

[14] J. Hirokawa and M. Ando, "Single-layer feed waveguide consisting of posts for plane TEM wave excitation in parallel plates," IEEE Transactions on Antennas and Propagation, vol. 46, no. 5, pp. 625630, 1998.

[15] D. Deslandes and W. Ke, "Accurate modeling, wave mechanisms, and design considerations of a substrate integrated waveguide," IEEE Transactions on Microwave Theory and Techniques, vol. 54, no. 6, pp. 2516-2526, 2006.

[16] X. Feng and W. Ke, "Guided-wave and leakage characteristics of substrate integrated waveguide," IEEE Transactions on Microwave Theory and Techniques, vol. 53, no. 1, pp. 66-73, 2005.

[17] M. Bozzi, M. Pasian, L. Perregrini, and W. Ke, "On the losses in substrate integrated waveguides," in European Microwave Conference, 2007, pp. 384-387.

[18] P. Sehyun, Y. Tsunemitsu, J. Hirokawa, and M. Ando, "Center feed single layer slotted waveguide array," IEEE Transactions on Antennas and Propagation, vol. 54, no. 5, pp. 1474-1480, 2006.

[19] Y. Miura, J. Hirokawa, M. Ando, Y. Shibuya, and G. Yoshida, "Doublelayer full-corporate-feed hollow-waveguide slot array antenna in the 60GHz band," IEEE Transactions on Antennas and Propagation, vol. 59, no. 8, pp. 2844-2851, 2011.

[20] A. Algaba Brazlez, "Gap waveguide for packaging microstrip filters \& investigation of transitions from planar technologies to ridge gap waveguide," Licentiate Thesis, 2013.

[21] E. Rajo-Iglesias, A. U. Zaman, and P. S. Kildal, "Parallel plate cavity mode suppression in microstrip circuit packages using a lid of nails," IEEE Microwave and Wireless Components Letters, vol. 20, no. 1, pp. 31-33, 2010.

[22] E. Pucci, A. U. Zaman, E. Rajo-Iglesias, P. S. Kildal, and A. A. Kishk, "Study of Q-factors of ridge and groove gap waveguide resonators," IET Microwaves, Antennas \& Propagation, vol. 7, no. 11, pp. $900-908$, 2013.
[23] H. Kirino and K. Ogawa, "A 76 GHz multi-layered phased array antenna using a non-metal contact metamaterial waveguide," IEEE Transactions on Antennas and Propagation, vol. 60, no. 2, pp. 840-853, 2012.

[24] A. U. Zaman, "Gap waveguide: Low loss microwave passive components and MMIC packaging technique for high frequency applications," $\mathrm{Ph} . \mathrm{D}$ dissertation, 2103.

[25] E. Pucci, E. Rajo-Iglesias, and P. S. Kildal, "Design of a dual-mode horn element for microstrip gap waveguide fed array," in Seventh European Conference on Antennas and Propagations, EuCAP 2013.

[26] A. D. Oliver, P. J. B. Clarricoats, A. A. Kishk, and L. Shafai, Microwave Horns and Feeds, 1st ed. IEEE Press, 1994.

[27] CST Microwave Studio. www.cst.com, 2009.

[28] P. S. Kildal, Foundations of Antennas: A Unified Approach. Studentlitteratur AB, 2000.

[29] Y.-W. Jang, "Wideband T-shaped microstrip-fed twin-slot array antenna," ETRI Journal, vol. 23, no. 1, 2001.

[30] A. A. Kishk, A. U. Zaman, and P. S. Kildal, "Numerical prepackaging with PMC lid - efficient and simple design procedure for microstrip circuits including the packaging," ACES Journal, vol. 27, no. 5, pp. 389-398, 2012.

[31] Advanced Design System (ADS). www.home.agilent.com, 2013.

[32] C. Gahete Arias, M. Baquero Escudero, A. Valero-Nogueira, and A. Vila Jimenez, "Test-fixture for suspended-strip gap-waveguide technology on Ka-band," accepted for publication in IEEE Microwave and Wireless Components Letters, 2013. 